\title{
Dermatologische Differentialdiagnosen von idiopathischen entzündlichen Muskelerkrankungen
}

\section{Dermatological Differential Diagnoses of Idiopathic Inflammatory Muscle Disease}

Autoren

Cord Sunderkötter ${ }^{1}$, Linda Golle ${ }^{1}$

Institute

1 Universitätsklinik und Poliklinik für Dermatologie und Venerologie, Universitätsklinikum Halle, Halle, Deutschland

Schlüsselwörter

Myositiden, Lupus erythematodes, Anti-Synthetase-

Syndrom, Dermatomyositis

Key words

dermatomyositis, Anti-synthetase syndrome, Lupus erythematosus

Bibliografie

Akt Rheumatol 2021; 46: 382-387

DOI $10.1055 / a-1551-5556$

ISSN 0341-051X

(c) 2021. Thieme. All rights reserved.

Georg Thieme Verlag KG, Rüdigerstraße 14,

70469 Stuttgart, Germany

Korrespondenzadresse

Cord Sunderkötter

Universitätsklinik und Poliklinik für Dermatologie und

Venerologie, Universitätsklinikum Halle

Ernst-Grube-Straße 40

06120 Halle

Deutschland

Tel.: 0345 5573925, Fax: 03455573942

cord.sunderkoetter@uk-halle.de

\section{ZUSAMMENFASSUNG}

Die Hautsymptome sind für die Differenzialdiagnose der Myositiden wichtig, da sie einmal entscheidend für die Unterscheidung der Dermatomyositis und des Anti-Synthetase-Syndroms von den anderen Autoimmun-Myositiden sind, und da sie bei Myositiden im Rahmen von Overlap Syndromen Hinweise auf die zugrunde liegenden Autoimmunerkrankungen (Lupus erythematodes, systemische Sklerose u. a.) geben können. Daher sollte bei Patienten mit einer Myositis eine genaue Inspektion der Haut erfolgen. Fast pathognomonisch für die Dermatomyositis ist die Trias aus symmetrischem, fliederfarbenem Erythem auf den Oberlidern (heliotropes Erythem), Erytheme oder flache Papeln oder Plaques über den proximalen interphalangealen und metacarpophalangealen Fingergelenken (so genannte Gottron Papeln) und Erytheme über Knien, Ellenbogen oder Knöcheln (so genanntes Gottron Zeichen). Daneben gibt es eine Reihe weiterer, typischer Hautsymptome (peitschenabdruckartige Erytheme, Poikilodermie, dystrophe Nagelhäutchen, Vaskulitiden, Juckreiz). Beim Anti-Synthetase-Syndrom treten neben einem Raynaud Phänomen als charakteristisches Symptom die sog. Mechanikerhände („mechanic's hands“) auf, d. h. Hyperkeratosen und Fissuren lateral an den Fingern. Klinisch und histologisch sind die Hautsymptome beim LE nicht eindeutig von denen der Dermatomyositis zu trennen, auch wenn es einige Unterschiede gibt. Die Hauteffloreszenzen sprechen nicht immer gut auf die Therapien der Myositis an. Ein Rückgang wurde unter Glukokortikoiden, Methotrexat, IVIG, Mycophenolatmofetil und Rituximab beobachtet. Als systemische Therapie gegen die Hautbeteiligung hat Hydroxychloroquin Wirksamkeit gezeigt, manchmal nur in Kombination mit Mepacrin. Zur zusätzlichen topischen Behandlung eignen sich Glukokortikoide und Calcineurin-Inhibitoren. Eine generelle Maßnahme ist der konsequente Sonnenschutz.

\section{ABSTRACT}

Skin symptoms are important in the differential diagnosis of myositides, as they are crucial in distinguishing dermatomyositis and anti-synthetase syndrome from other autoimmune myositides, and, in the context setting of overlap syndromesthey provide clues to potentially underlying autoimmune diseases (e. g. lupus erythematosus, systemic sclerosis). Therefore, careful inspection of the skin should be performed in all patients with myositis. The triad of symmetrical, lilac erythema on the upper eyelids (heliotropic erythema), erythema or flat papules or plaques over the proximal interphalangeal and metacarpophalangeal digital joints (so-called Gottron papules) and scaly erythema over the knees, elbows, or ankles (so-called Gottron sign) are almost pathognomic for dermatomyositis. In addition, there are other, sometimes rare, but nevertheless typical symptoms (flaggelate erythema, poikiloderma, dystrophic cuticles, vasculitides, pruritus). In anti-synthetase syndrome, a characteristic symptom are the so-called mechanic's hands, i. e. hyperkeratosis and fissures, mainly on the lateral aspect of the fingers. Clinically and histologically, several skin 
symptoms in LE are similar and cannot always be clearly distinguished from those in dermatomyositis, but there are some differences, which, if present, are helpful in differential diagnosis. Cutaneous involvement in dermatomyositis does not always respond well to systemic therapies of myositis. Some of them may be efficacious (glucocorticoids, methotrexate, IVIG, mycophenolate mofetil, rituximab). For persistent or predominant skin lesions, hydroxychloroquine has been shown to be helpful, sometimes only in combination with quinacrine. For additional topical treatment, glucocorticoids and calcineurin inhibitors can be recommended. A general measure is consistent protection from UV light.
Können Hautsymptome bei der Differenzialdiagnose der Myositiden hilfreich sein? Ja, indem sie einmal entscheidend - und namensgebend - für die Unterscheidung der Dermatomyositis (und des neuerdings als eigene Entität betrachteten Anti-Synthetase-Syndroms) von den anderen Autoimmun-Myositiden sind, und indem sie bei Myositiden im Rahmen immunologischer Systemerkrankungen (Overlap-Syndrome) Hinweise auf die jeweils zugrunde liegenden Autoimmunerkrankungen geben können, also auf einen Lupus erythematodes (LE), eine systemische Sklerose (SSc), eine Mischkollagenose, ein Sjögren-Syndrom, eine rheumatoide Arthritis oder eine Begleitmyositis bei systemischen Vaskulitiden [1]. Diese Übersichtsarbeit möchte auf die jeweils wichtigsten Effloreszenzen hinweisen.

\section{Hautsymptome bei DM und bei Anti-Synthetase-Syndrom}

Die Dermatomyositis galt lange Zeit als einzige Autoimmun-Myositis, die mit Hauteffloreszenzen einhergeht. Wenn man aktuell das Anti-Synthetase-Syndrom nicht als eine Untergruppe der DM, sondern als eigene Entität in der Gruppe der Autoimmun-Myositiden betrachtet, dann wären es jetzt diese beide Autoimmun-Myositiden, die Hautsymptome aufweisen.

Wenn eine proximale, symmetrische Muskelschwäche (mit erhaltenen Eigenreflexen) mit den (unten genannten) für die Dermatomyositis charakteristischen Hauterscheinungen einhergeht, dann kann die Diagnose Dermatomyositis sogar erst einmal gestellt werden, ohne dass zusätzliche eine Muskelbiopsie vorliegt. Letztere wird, gerade wenn nicht nur die typischen Hautsymptome vorliegen (auch zum Ausschluss einer Overlap-Myositis) zur Absicherung und genaueren Differenzialdiagnostik meist noch durchgeführt und in den Leitlinien empfohlen [2].

Welches sind die charakteristischen Hauterscheinungen? Häufig und fast pathognomonisch ist folgende Trias, wenn auch ihre drei Komponenten initial in unterschiedlichem Ausmaß vorhanden sind:

1. ein symmetrisches, rot bis blau-violett-farbenes Erythem auf den Oberlidern, oft gepaart mit leichten bis mäßig starken periorbitalen Ödemen - das heliotrope Erythem ( $\bullet$ Abb. 1)

2. Erytheme oder flache Papeln oder Plaques, oft schuppend, über den proximalen interphalangealen und metacarpophalangealen Fingergelenken (so genannte Gottron Papeln, siehe - Abb. 2);

3. schuppende Erytheme über Knien, Ellenbogen oder Knöcheln (so genanntes Gottron Zeichen).
Das heliotrope Erythem ist typisch für den akuten Schub [2]. Es wird meist begleitet von einem rotlividen Erythem oder leicht erhabenen Plaques auf beiden Wangen und auf der Nase, also einem „Schmetterlingserythem“ wie beim Lupus erythematodes, und ähnlichen Erythemen im Dekolleté und Nacken (das so genannte „Schal“-Zeichen, siehe - Abb. 3) sowie auf den Streckseiten der Extremitäten einschließlich der Handrücken, mithin auf Hautarealen, die dem Sonnenlicht ausgesetzt sind. Oft befindet sich auf den Erythemen eine leichte Schuppung. Mit zunehmender Dauer der $\mathrm{Er}$ krankung entwickeln sich in den betroffenen Stellen Hyper- und Hypopigmentierungen, Teleangiektasien sowie eine Verdünnung (Atrophie) der Epidermis (Poikilodermie).

Weitere überaus typische, aber eher seltene Hautsymptome sind:

- streifige Geißel- oder peitschenabdruckartige Erytheme (in der angloamerikanischen Terminologie „flagellate erythema“),

- eine (weitere) Poikilodermie am seitlichen Oberschenkel (in der angloamerikanischen Terminologie wegen dieser bezeichnenden Lokalisation das "holster sign”, also Pistolenhalfter-Zeichen) ( $\triangleright$ Abb. 4),

- das dystrophe Nagelhäutchen, welches beim Zurückschieben schmerzt (Keining-Zeichen)

- ein schuppendes Erythem und ein ausgeprägter Juckreiz auf der behaarten Kopfhaut (Capillitium)

Typisch für die Dermatomyositis, aber in ähnlicher Weise auch für SLE oder andere Autoimmun-Kollagenosen sind:

- eine Rötung des Nagelfalzes ( $\triangleright$ Abb. 5) mit erweiterten Kapillaren und entsprechend mit kapillarmikroskopischen Auffälligkeiten, - sie findet sich auch bei SLE und häufig bei SSc (hier mit sehr typischen kapillarmikroskopischen Befunden, die bei DM nicht ganz so pathognomonisch sind) [3]

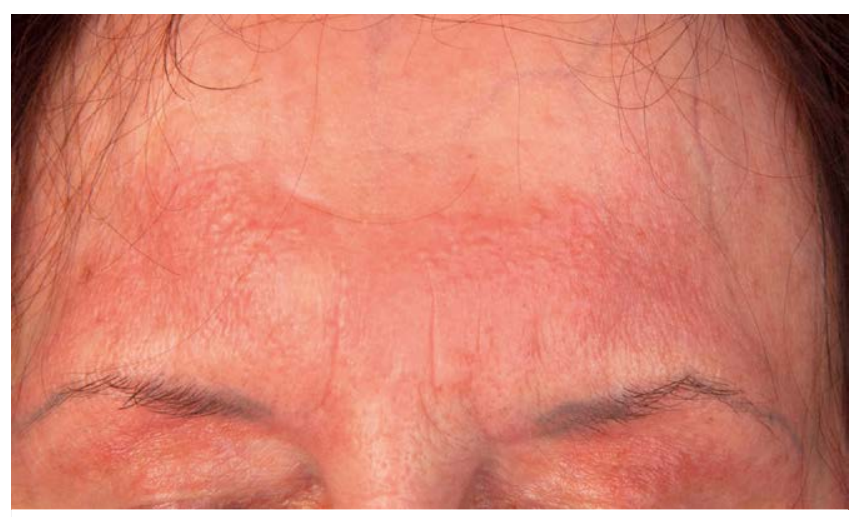

Abb. 1 heliotropes Erythem. 


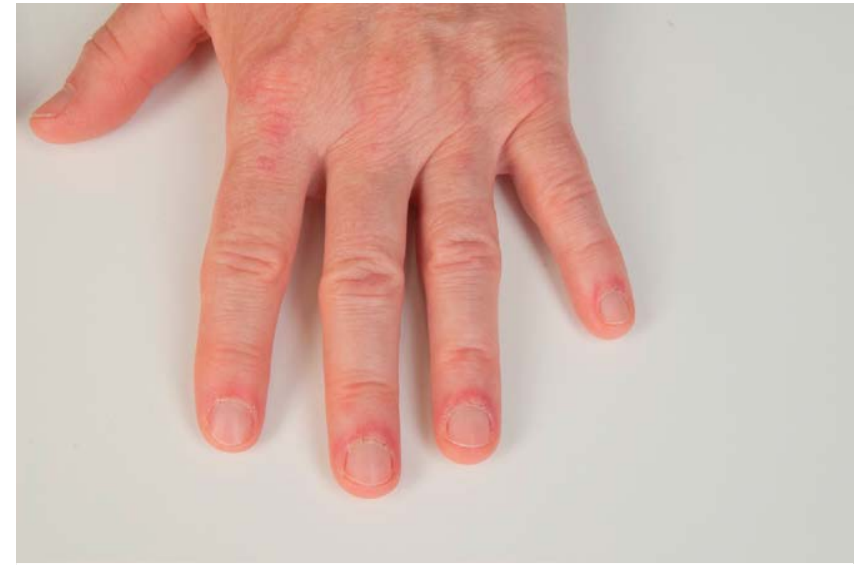

- Abb. 2 „Gottron'sche Papeln“ über den dorsalen proximalen interphalangealen und metacarpophalangealen Fingergelenken.

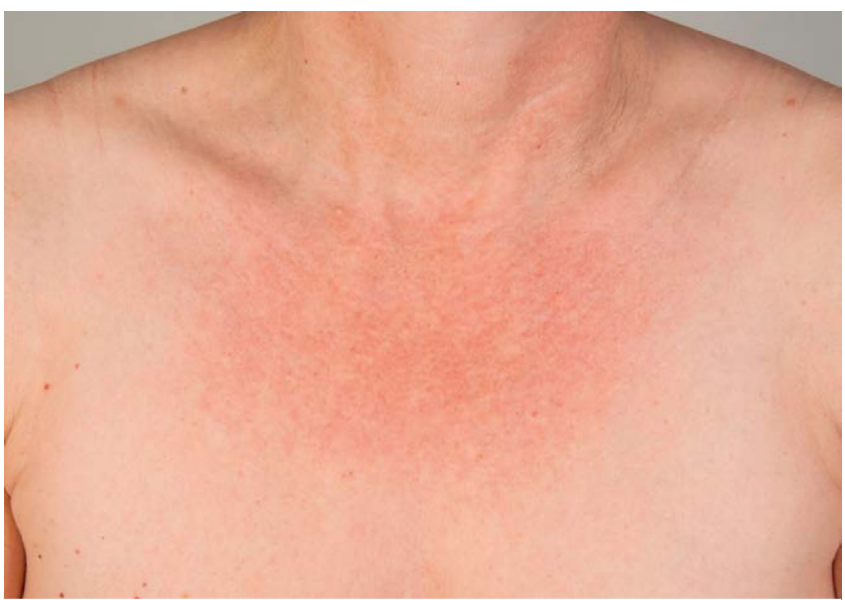

- Abb. 3 "Schal-Zeichen“.

- eine Entzündung in der Tiefe des Fettgewebes (Pannikulitis), die sich oft am Oberschenkel abspielt und nachfolgend zu Einbuchtungen im Gewebe aufgrund einer Lipoatrophie führt (eine Entzündung des Fettgewebes findet sich auch typischerweise bei SLE (Lupus-Pannikulitis), dort nicht ganz so häufig auf den Oberschenkel begrenzt)

- eine schuppende Rötung der gesamten Haut (exfoliative Erythrodermie),

- Telangiektasien am Zahnfleisch

- Pusteln an Ellenbogen und Knien

- eine Vaskulitis der kleinen Gefäße [4] (v. a. bei juveniler DM),

- Kalkablagerungen in der Haut (Calcinosis) (auch v. a. bei juveniler DM)

- eine nicht vernarbende Alopezie

- eine erhöhte Sonnenlichtempfindlichkeit (Photosensitivität), die sich auch schon in der Prädilektion der Herde ausspricht und sehr typisch auch für den LE ist

- Erosionen der Haut oder gar Ulzerationen - sie finden sich oft bei Malignom-assoziierter DM

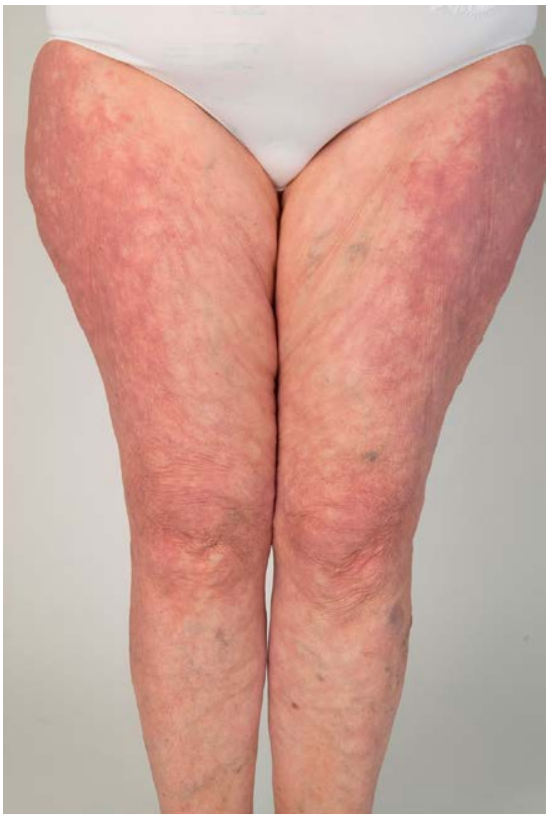

- Abb. 4 Halfterzeichen („holster sign“) an den seitlichen Oberschenkeln und Livedozeichnung.

- ein Raynaud Phänomen - es findet sich insbesondere bei Vorliegen von anti-SRP-Antikörpern oder anti-Synthetase Autoantikörpern, sowie bei juveniler DM auf und bei Sklerodermie- Polymyositis Overlap Syndrom.

Bei dunkelhäutigen Menschen sind die erythematösen (oft akuten) Hautsymptome schlechter, die chronischen Zeichen der Schädigung dagegen oft besser zu erkennen als bei Kaukasiern [5].

An subjektiven Symptomen kann gerade bei der DM ein ausgeprägter hartnäckiger und quälender Juckreiz die Hautsymptome bei der DM begleiten, bevorzugt auf der behaarten Kopfhaut, aber auch flächig auf der gesamten Haut.

Beim Anti-Synthetase-Syndrom tritt neben dem Raynaud Phänomen noch ein charakteristisches Symptom auf, dass man sonst bei der DM, oder bei anderen Al-Dermatosen mit Myositis nicht so häufig sieht, allenfalls bei den Fällen mit MDA 5-Antikörpern [6], nämlich die sogenannten Mechanikerhände („mechanic's hands“, siehe $\mathbf{A b b . 6 )}$, d. h. Hyperkeratosen und Fissuren oder Rhagaden lateral an den Fingern.

Besonders ausgeprägte oder therapierefraktäre Effloreszenzen, Hautnekrosen, ein starker Juckreiz (oft zusammen mit positiven Titern von TIF-1Y-Antikörpern) sind ein Hinweis auf einen mit Dermatomyositis assoziierten malignen Tumor $[2,6]$.

Mit zunehmend häufigerer Bestimmung der Myositis-spezifischen Antikörper wird sich in den folgenden Jahren zeigen, welche Konstellation an Hautsymptomen mit bestimmten Autoanitkörpern assoziiert ist und auf bestimmte Verläufe verweist [7].

In einigen Fällen (5 bis ca. 20\%) treten die genannten typischen Hautsymptome und einem mit DM vereinbaren histologischen Befund (bei gleichzeitigem Ausschluss eines LE) hingegen auch ganz ohne eine erkennbare klinische und laborchemische Muskelbeteiligung (keine Muskelschwäche, keine erhöhten Muskelenzyme) auf [8-10]. 


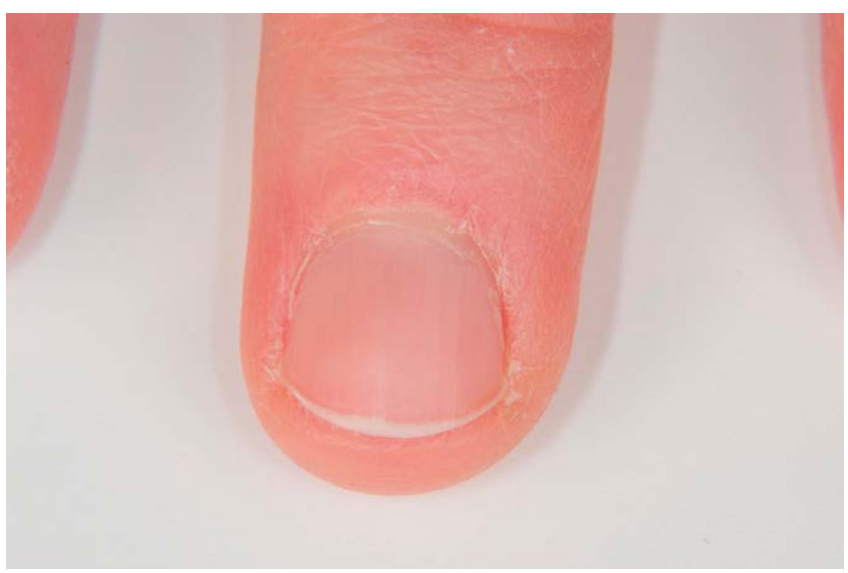

- Abb. 5 Geröteter, hyperkeratotischer Nagelfalz.

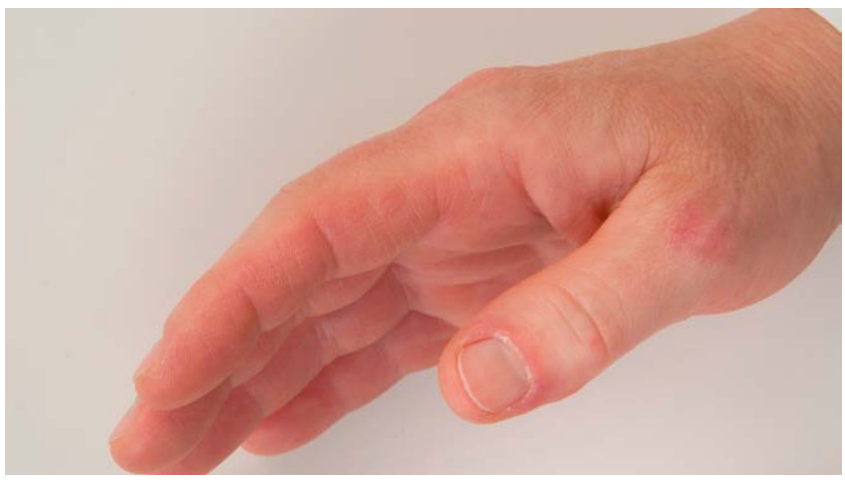

- Abb. 6 Mechanikerhände („mechanic’s hands“).

Ein Teil dieser Patienten mag in der Magnetresonanztomographie (MRT) Entzündungsherde aufweisen und in einer entsprechend gesteuerten Muskelbiopsie Zeichen einer Myositis [11], aber bei mehr als der Hälfte dieser Patienten fehlt auch ein auffälliger MRT-Befund. Man spricht dann von einer amyopathischen DM oder „DM sine myositis“. Bei Patienten mit dieser Ausschlussdiagnose sollte für mindestens 2 Jahre regelmäßig (alle 2-3 Monate) nach Muskelbeteiligung gefahndet werden (Zeichen für Muskelschwäche oder pathologische Serumspiegel für Muskelenzyme, ggf. MRT der potentiell befallenen Muskeln und bei Entzündungszeichen Muskelbiopsie) [2]. Nach 2 Jahren wird eine Myositis unwahrscheinlicher [12]. Mitunter ist, verglichen mit den Hautsymptomen, nur eine vergleichsweise geringe Muskelschwäche vorhanden, so dass diese Patienten mit Dermatomyositis eher wegen der im Gesicht auffälligen und störenden Erytheme zum Haus- oder Hautarzt gehen und nicht wegen der Muskelbeschwerden.

\section{Hautsymptome und Unterschiede bei Overlap-Syndromen}

Während bei der klassischen Dermatomyositis die Hautsymptome der Myositis sowohl vorangehen oder mit oder kurz nach ihrem Beginn erscheinen, können sie bei den Overlap-Myositiden sowohl fehlen als auch verspätet auftreten [7].
Mithin spricht ihr Fehlen bei Symptomen einer Myositis zwar eindeutig gegen eine Dermatomyositis, aber nicht unbedingt gegen eine Overlap-Kollagenose mit Anteilen einer DM. Wenn bei den Overlapsyndromen das typische heliotrope Erythem oder die Gottronschen Papeln auftreten, sind sie sind meist weniger ausgebreitet und transienter als bei der reinen Dermatomyositis [7].

Außerdem treten bei den Overlap Myositiden zusätzlich die Hautsymptome der jeweiligen überlappenden Kollagenose auf wie z. B. geschwollen Finger (sog. „puffy hands“) und später Sklerodakytlie und digitale Ulzerationen bei der DM-SSc, keratotische oder erythematöse, randbetont squamös-keratotische Plaques, orale oder nasale Erosionen oder Ulzera bei SLE, anhaltende Augenund Mundtrockenheit und manchmal makuläre Vaskulitis (Purpura benigna Waldenströn) bei Sjögren Syndrom [1].

Klinisch ist wegen der ähnlichen Hautsymptome oft die Unterscheidung zwischen Dermatomyositis und LE schwierig. Hierzu sind zwei Feststellungen wichtig und merkenswert: a) eine eindeutige klinische Abgrenzung der Erytheme zwischen Dermatomyositis und Lupus erythematodes ist weder klinisch noch histologisch möglich, zumal sich DM und SLE auch in den anderen Effloreszenzen ähneln; b) es gibt Unterschiede, die aber aufgrund der obigen Feststellung alleine nicht zur Differenzialdiagnose ausreichen, jedoch bedeutsame Indizien sind.

Das Erythem bei DM ist im Unterschied zum SLE meist mehr livide und weniger rötlich, nicht so scharf abgesetzt, das periorbitale heliotrope Zeichen und die Ausdehnung auf die Streckseiten sind deutlicher ausgeprägt, Juckreiz häufiger, und auf den Fingerstreckseiten finden sich die Erytheme über den Gelenken (Gottron Papeln), während sie sich beim SLE auffälligerweise oft über den Fingergliedern zwischen den Gelenken befinden.

Weitere Differenzialdiagnosen bei einem Erythem mit Betonung der Gesichtsmitte sind periorale Dermatitis, seborrhoisches Ekzem und Rosazea. Bei diesen Dermatosen ist aber im Gegensatz zu DM und SLE der nasolabiale Bereich deutlich befallen.

Bei Herden auf dem Kopf sollte ohne klare muskuläre Symptomatisch auch an ein seborrhoisches Ekzem und bei zusätzlichem Befall der Streckseiten der Extremitäten zudem an eine Psoriasis gedacht werden.

Wegen möglicher Differenzialdiagnosen sollte eine Hautbiopsie entnommen werden. Die mit einer Dermatomyositis vereinbaren, aber nicht pathognomonischen und auch bei LE auffindbaren Zeichen sind Atrophie der Epidermis, Degeneration der Basalmembranzone mit vakuolisierten basalen Keratinozyten, subepidermales, oft spärliches lymphozytäres Infiltrat (sog. Interfacedermatitis) und interstitielle Muzineinlagerungen in der Dermis. Die sog. Gottronschen Papeln zeigen eher eine Akanthose als Atrophie der Epidermis.

\section{Therapie der Hautsymptome}

Die meisten Hauteffloreszenzen sprechen auf einige, aber nicht alle systemischen Therapien der Myositis an. Oft bleiben die Hautsymptome sowie der beeinträchtigende Juckreiz bestehen, nachdem die Myositis bereits angesprochen hat.

Von den zur Behandlung der Myositis eingesetzten Wirkstoffen ist für folgende eine Wirksamkeit auch gegen die Hauterscheinungen nachgewiesen worden: Glukokortikoide, Methotrexat [13], IVIG [14, 15], Mycophenolatmofetil [16], Rituximab [17] und Stammzelltransplantation [18]. Azathioprin kann zwar eine Wirk- 
samkeit gegen Hautsymptome aufweisen, wird aber eher meist nur noch zusätzlich als Steroid-sparendes Medikament eingesetzt.

Wenn nur die typischen Hautsymptome einer DM mit entsprechendem histopathologischen Befund vorliegen, aber (noch) keine Muskelbeteiligung, sollte zunächst keine immunsuppressive systemische Therapie begonnen werden.

Antimalariamittel (z. B. Hydroxychloroquin (Quensyl) $200 \mathrm{mg}$, 1-2x täglich) sind wirksam nicht nur gegen die amyopathische DM, sondern auch bei Hautsymptomen, die auf vorher angesetzte immunsuppressive Therapien nicht angesprochen haben (wohingegen Hydroxycloroquin keine sichere Wirksamkeit auf die Myositis hat [19]). Die Wirksamkeit kann - und sollte bei ungenügender Wirkung - durch Kombination von Hydroxychloroquin oder Chloroquin mit Mepacrin (100 mg/d) verbessert werden [20].

Es muss angemerkt werden, dass Hydroxychloroquin bei Patienten mit DM häufiger zu kutanen Arzneimittelreaktion führt (ca $20 \%$ ), allerdings vor allem bei Patienten mit anti-SAE-1/2 Autoantikörpern und offenbar dagegen kaum bei anti-MDA-5 Autoantikörpern [21].

Zur zusätzlichen topischen Behandlung der Hauteffloreszenzen stehen Glukokortikoide zur Verfügung, die auch den heftigen Juckreiz vermindern können. Nach gutem Ansprechen kann, zur Vermeidung von Atrophien oder anderer Nebenwirkungen für die Erhaltungstherapie ein topischer Calcineurin-Inhibitor (Tacrolimus $0.1 \%$ ) versucht werden [22], allerdings außerhalb der Zulassung (Off-label).

Eine generelle Maßnahme zur Prophylaxe der Hautbeteiligung und Unterstützung der Therapie ist aufgrund der erhöhten UVEmpfindlichkeit der konsequente Sonnenschutz mit Textilien und Sonnenschutzcremes. Letztere sollten einen hohen Lichtschutzfaktor haben, müssen aber vor allem in ausreichender Mengen aufgetragen werden, da ihr Schutz nicht linear sondern gar exponentiell fällt, wenn die Auftragsmenge nicht mindestens $2 \mathrm{mg} / \mathrm{cm}^{2}$ beträgt (entspräche bei den üblichen $2 \mathrm{~m}^{2}$ Körperoberfläche $40 \mathrm{~g}$, also oft einer halben Tube für das gesamte Integument).

Gegen die bei Kindern häufig auftretende, aber auch im Erwachsenenalter vorkommende, kutane Kalzinose müssten kleinere Herde (von $\leq 2$ mm Durchmesser) analog zur Kalzinose bei SSc gut auf topisches Natriumthiosulfat $10 \%$ [23] ansprechen (im so genannten off-label Einsatz). Bei größeren oder tiefer gelegenen Läsionen kann eine subkutane oder intravenöse Verabreichung von Natriumthiosulfat erwogen werden, zu der wir bei Erwachsenen mit SSc gerade gute Erfahrung machen, die aber bei Kindern bislang nicht erprobt ist. Außerdem mögen Kalzinosen auf Diltiazem (5 mg/kg/Tag) und Pamidronat (4 mg/kg/Tag) [24] ansprechen und durch systemische Glukokortikoide verhinderbar sein [25].

\section{Fazit}

Da die Hautsymptome ein entscheidendes Kriterium für die DM darstellen, ist bei allen Patienten mit einer Myositis eine genaue Inspektion der Haut einschließlich des behaarten Kopfes, der Nägel und Nagelfalze erforderlich. Fast pathognomonisch für die Dermatomyositis ist die Trias aus heliotropem Erythem, flachen Papeln über den proximalen Fingergelenken und Erythemen über Knien, Ellenbogen oder Knöcheln.
Interessenkonflikt

Die Autorinnen/Autoren geben an, dass kein Interessenkonflikt besteht.

Literatur

[1] Sunderkötter CH, Zelger B, Chen KR et al. Nomenclature of Cutaneous Vasculitis: Dermatologic Addendum to the 2012 Revised International Chapel Hill Consensus Conference Nomenclature of Vasculitides. Arthritis Rheumatol 2018; 70: 171-184

[2] Sunderkötter C, Nast A, Worm M et al. Guidelines on dermatomyositis-excerpt from the interdisciplinary $\mathrm{S} 2 \mathrm{k}$ guidelines on myositis syndromes by the German Society of Neurology. J Dtsch Dermatol Ges 2016; 14: 321-338

[3] Klein-Weigel PF, Sunderkötter C, Sander O. Nailfold capillaroscopy microscopy-an interdisciplinary appraisal. Vasa 2016; 45: 353-364

[4] Sunderkötter C, Lamprecht P, Mahr A et al. Nomenklatur der kutanen Vaskulitiden-deutschsprachige Definitionen des Dermatologischen Anhanges zur Chapel Hill Consensus Conference. J Dtsch Dermatol Ges 2018; 16 : 1425-1433

[5] Schlecht N, Sunderkötter C, Niehaus S, Nashan D. Adulte Form der Dermatomyositis-ein Update. J Dtsch Dermatol Ges 2020; 18: 995-1014

[6] Ogawa-Momohara M, Kinoshita F, Muro Y et al. Autoantibody profiles in patients' sera associated with distribution patterns of dermatomyositis skin symptoms. J Am Acad Dermatol 2021; 84: 1720-1722

[7] Troyanov Y, Targoff IN, Payette MP et al. Redefining dermatomyositis: a description of new diagnostic criteria that differentiate pure dermatomyositis from overlap myositis with dermatomyositis features. Medicine (Baltimore) 2014; 93: 318-332. Erratum in: Medicine (Baltimore). 201493:414

[8] el-Azhary RA, Pakzad SY. Amyopathic dermatomyositis: retrospective review of 37 cases. J Am Acad Dermatol 2002; 46: 560-565

[9] Gerami P, Schope JM, McDonald L et al. A systematic review of adult-onset clinically amyopathic dermatomyositis (dermatomyositis siné myositis): a missing link within the spectrum of the idiopathic inflammatory myopathies. J Am Acad Dermatol 2006; 54: 597-613

[10] Bendewald MJ, Wetter DA, Li X et al. Incidence of dermatomyositis and clinically amyopathic dermatomyositis: a population-based study in Olmsted County, Minnesota. Arch Dermatol 2010; 146: 26-30

[11] Lam WW, Chan $\mathrm{H}$, Chan YL et al. MR imaging in amyopathic dermatomyositis. Acta Radiol 1999; 40: 69-72

[12] Rockerbie NR, Woo TY, Callen JP et al. Cutaneous changes of dermatomyositis precede muscle weakness. J Am Acad Dermatol 1989; 20: 629-632

[13] Kasteler JS, Callen JP. Low-dose methotrexate administered weekly is an effective corticosteroid-sparing agent for the treatment of the cutaneous manifestations of dermatomyositis. J Am Acad Dermatol 1997; 36: $67-71$

[14] Dalakas MC, Illa I, Dambrosia JM et al. A controlled trial of high-dose intravenous immune globulin infusions as treatment for dermatomyositis. N Engl J Med 1993; 329: 1993-2000

[15] Aggarwal R, Charles-Schoeman C, Schessl J et al. Prospective, double-blind, randomized, placebo-controlled phase III study evaluating efficacy and safety of octagam $10 \%$ in patients with dermatomyositis („ProDERM Study“). Medicine (Baltimore) 2021; 100 : e23677

[16] Edge JC, Outland JD, Dempsey JR et al. Mycophenolate mofetil as an effective corticosteroid-sparing therapy for recalcitrant dermatomyositis. Arch Dermatol 2006; 142: 65-69 
[17] Oddis CV, Reed AM, Aggarwal R et al. Rituximab in the treatment of refractory adult and juvenile dermatomyositis and adult polymyositis: a randomized, placebo-phase trial. Arthritis Rheum 2013; 65: 314-324

[18] Wang D, Zhang H, Cao M et al. Efficacy of allogeneic mesenchymal stem cell transplantation in patients with drug-resistant polymyositis and dermatomyositis. Ann Rheum Dis. 2011; 70: 1285-1288

[19] Woo TY, Callen JP, Voorhees JJ et al. Cutaneous lesions of dermatomyositis are improved by hydroxychloroquine. J Am Acad Dermatol 1984; 10: 592-600

[20] Ang GC, Werth VP. Combination antimalarials in the treatment of cutaneous dermatomyositis: a retrospective study. Arch Dermatol 2005; 141: 855-859

[21] Wolstencroft PW, Casciola-Rosen L, Fiorentino DF. Association Between Autoantibody Phenotype and Cutaneous Adverse Reactions to Hydroxychloroquine in Dermatomyositis. JAMA Dermatol 2018; 154: $1199-1203$
[22] Hollar CB, Jorizzo JL. Topical tacrolimus $0.1 \%$ ointment for refractory skin disease in dermatomyositis: a pilot study. J Dermatolog Treat 2004; 15: 35-39

[23] von Hodenberg C, Neufeld M, Wohlrab J et al. Sichere topische Behandlung der digitalen Calcinosis cutis mit Natriumthiosulfat-Fallserie mit sechs Patienten. J Dtsch Dermatol Ges 2020; 18: 1181-1183

[24] Oliveri MB, Palermo R, Mautalen C et al. Regression of calcinosis during diltiazem treatment in juvenile dermatomyositis. J Rheumatol 1996; 23: 2152-2155

[25] Paller AS. The use of pulse corticosteroid therapy for juvenile dermatomyositis. Pediatr Dermatol 1996; 13: 347-348 